\title{
Arteriovenous malformation embocure score: AVMES
}

${ }^{1}$ Department of Neurological Surgery, Rush University Medical Center, Chicago, Illinois, USA

${ }^{2}$ Rush University Medical College, Rush University Medical Center, Chicago, Illinois, USA

\section{Correspondence to} Dr Demetrius K Lopes, Department of Neurological Surgery, Rush University Medical Center, 1725 W Harrison St, Professional Building Suite 855, Chicago, IL 60612, USA;

Demetrius_Lopes@Rush.edu

Received 11 April 2015 Revised 27 May 2015 Accepted 29 May 2015 Published Online First 15 June 2015

\section{CrossMark}

To cite: Lopes DK Moftakhar R, Straus D, et al. J Neurolntervent Surg 2016;8:685-691.

\author{
Demetrius K Lopes, ${ }^{1}$ Roham Moftakhar, ${ }^{1}$ David Straus, ${ }^{1}$ Stephan A Munich, ${ }^{1}$ \\ Fahad Chaus, ${ }^{2}$ Megan C Kaszuba ${ }^{2}$
}

\begin{abstract}
Background Cerebral arteriovenous malformations (CAVMs) may be treated with microsurgery, radiosurgery, endovascular surgery, or a combination of these modalities. Grading scales are available to aid the assessment of curative risk for microsurgery and radiosurgery. No grading system has been developed to assess the curative risk of endovascular surgery.

Objective To report our retrospective application of the AVM embocure score to patients treated at our institution between 2005 and 2011
\end{abstract}

Methods We performed a retrospective review of 39 patients with CAVM treated at our institution between 2005 and 2011 with the primary aim of achieving a curative embolization. After reviewing all the different variables associated with the conventional Onyx embolization technique for CAVMs, we identified the following as the most relevant characteristics influencing the chances for complete angiographic embolization and complication risk: the number of arterial pedicles and draining veins, size of AVM nidus, and vascular eloquence. We sought to develop a scoring system to assess the complication risk for a curative embolization of CAVM with liquid embolic Onyx (Covidien, Irvine, California, USA). We developed the AVM embocure score (AVMES). This scoring system ranges from 3 to 10 and is the arithmetic sum of the number of arterial pedicles feeding the AVM $(\leq 3,4-6,>6)$, the number of draining veins $(\leq 3,4-6,>6)$, the size of the AVM nidus in centimeters $(\leq 3,4-6,>6)$, and the vascular eloquence $(0-1)$. We applied AVMES to the same cohort of patients and validated the predictability of complete angiographic embolization and expected clinical risk of complication.

Results In lesions with an AVMES of $3(n=8)$, there was a $100 \%$ rate of complete AVM obliteration and $0 \%$ rate of major complications. In AVMES 4 ( $n=12)$ lesions, there was $75 \%$ complete obliteration rate, with $8 \%$ major morbidity. In AVMES $5(n=9)$ lesions, there was $78 \%$ complete obliteration and $11 \%$ major morbidity. In AVMES $>5(n=10)$ there was $20 \%$ complete obliteration and $30 \%$ major morbidity. Receiver-operator curve analysis showed that this scoring system was robust in its discriminative ability, with an area under the curve (AUC) of 0.8356 for complete obliteration without complication, $A U C=0.8240$ for complete obliteration regardless of the presence of major morbidity, and $\mathrm{AUC}=0.7529$ for major morbidity.

Conclusions The AVMES complements existing scoring systems for microsurgery and radiosurgery. It provides a valuable tool for risk assessment during the complex decision-making process in treating AVMs that accounts for angioarchitectural features of particular relevance to endovascular surgeons.

\section{INTRODUCTION}

Management of cerebral arteriovenous malformations is often a complex, therapeutic decision. Grading systems that help to predict the risk of curative treatment are important to assist this process, Since its introduction in 1986, the Spetzler-Martin scale $^{1}$ has been the most widely used grading system. It provides a validated and easily applied system to predict the microsurgical morbidity and mortality for various AVMs using readily available data (size, location, and venous drainage). The Spetzler-Martin scale, however, was developed exclusively in patients treated with microsurgical resection. The advent of new treatment modalities - radiosurgery and embolization-has enhanced our ability to effectively treat AVMs, and improved the safety of our interventions. However, the physical mechanism, technical challenges, limitations, and risks, vary significantly between microsurgery, radiosurgery, and embolization. ${ }^{2-7}$ Thus, the appropriateness of using the Spetzler-Martin scale for radiosurgery and embolization is uncertain and its use as a prognostic tool in these treatment modalities remains speculative. ${ }^{28} 9$ Recognizing these limitations, Pollock and Flickinger, ${ }^{10}$ have described a radiosurgery-based AVM scoring system. This system sought to separate patients into groups with varying likelihoods of achieving an "excellent" outcome (nidus obliteration without new neurologic deficit) using factors that were felt to be particularly relevant to radiosurgical treatment outcomes (AVM volume, patient age, and AVM location). The resultant AVM score was able to discriminate between patients in the best group (score $<0.75$ ), all of whom had excellent outcomes, and those in the worst group (score $>2.00$ ), where only 39\% of patients had excellent outcomes.

To date, however, there exists no prognostic tool that can be reliably applied to endovascular embolization of AVMs with the intention of complete obliteration. The ability to select the treatment modality that offers the best chance for successful treatment and the most favorable risk profile for a particular AVM requires our knowledge of these features for all therapeutic modalities. To this end, we sought to develop a readily applicable scoring system to aid in risk-stratifying AVMs for endovascular embolization. Here, we report our retrospective application of the AVM embocure score (AVMES) to patients treated at our institution between 2005 and 2011.

\section{PATIENTS AND METHODS}

\section{Patient selection and data collection}

Patients treated at our institution between 2005 and 2011 were included if they had a cerebral AVM that 


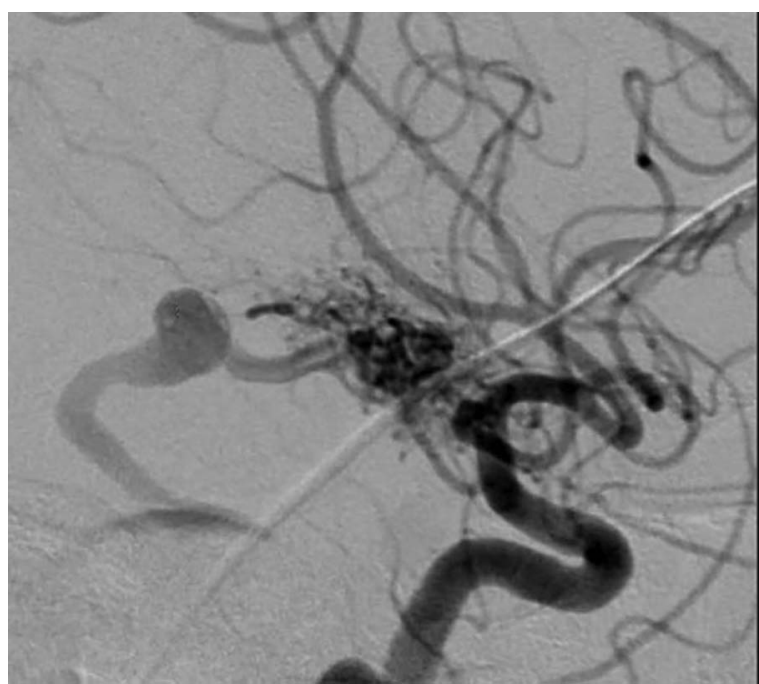

Figure 1 Example of grades of vascular eloquence. An arteriovenous malformation (AVM) was deemed eloquent if the arterial pedicle was $<20 \mathrm{~mm}$ from the internal carotid artery or the first segment of cerebral arteries (eg, A1, M1, P1 segments) or too small for microcatheterization. The assignment of non-eloquence was given if an AVM did not meet eloquent criteria.

had not been previously treated and underwent endovascular embolization with Onyx (Covidien, Irvine, California, USA) with curative intent during the study period. Exclusion criteria were prior treatment (microsurgery, radiosurgery, or embolization), planned staged multimodal treatment (microsurgery or radiosurgery), or lack of pre-embolization angiogram or medical records.
We retrospectively reviewed the medical records of these patients to obtain relevant information about their presentation, outcome, and procedure. Specifically, we collected patient age at the time of the procedure, sex, presenting symptoms, preoperative neurologic examination, number of embolization procedures performed, volume of Onyx injected, technical complications, access-related complications, new neurologic deficits (classified as minor if transient ( $<30$ days) or major if persistent), and whether the AVM was completely obliterated after embolization. AVM obliteration and embocure was considered only after MRI/MRA with contrast and DSA at the 6-month follow-up after embolization had shown no nidus and early draining vein.

\section{AVM embocure score}

The decision to consider embolization for curative treatment is intuitive and highly dependent on the surgeon's experience. In an attempt to standardize the endovascular approach to AVM embolization, we reviewed the angioarchitecture of AVMs treated with Onyx with the intention to cure. Subsequently, we identified four variables that were relevant to the successful curative embolization of AVM without clinical complications. These included the vascular eloquence of the arterial feeders (figure 1), number of arterial pedicles (figure 2), size of the AVM (figure 3), and the number of draining veins (figure 4). An AVM was considered to possess vascular eloquence if the arterial pedicle was $<20 \mathrm{~mm}$ from the internal carotid artery or the first segment of cerebral arteries (eg, A1, M1, P1 segments; figure 1) or too small for microcatheterization. We considered this to be vascular eloquence because iatrogenic occlusion or injury at these segments would result in major neurologic morbidity.
Figure 2 Example of grade of arterial pedicles. An arteriovenous malformation was given an arterial pedicle grade of 1 if $1-3$ arterial pedicles were identified as the arterial supply (A), 2 if $4-6$ pedicles were identified (B), and 3 if $>6$ pedicles were identified (C).
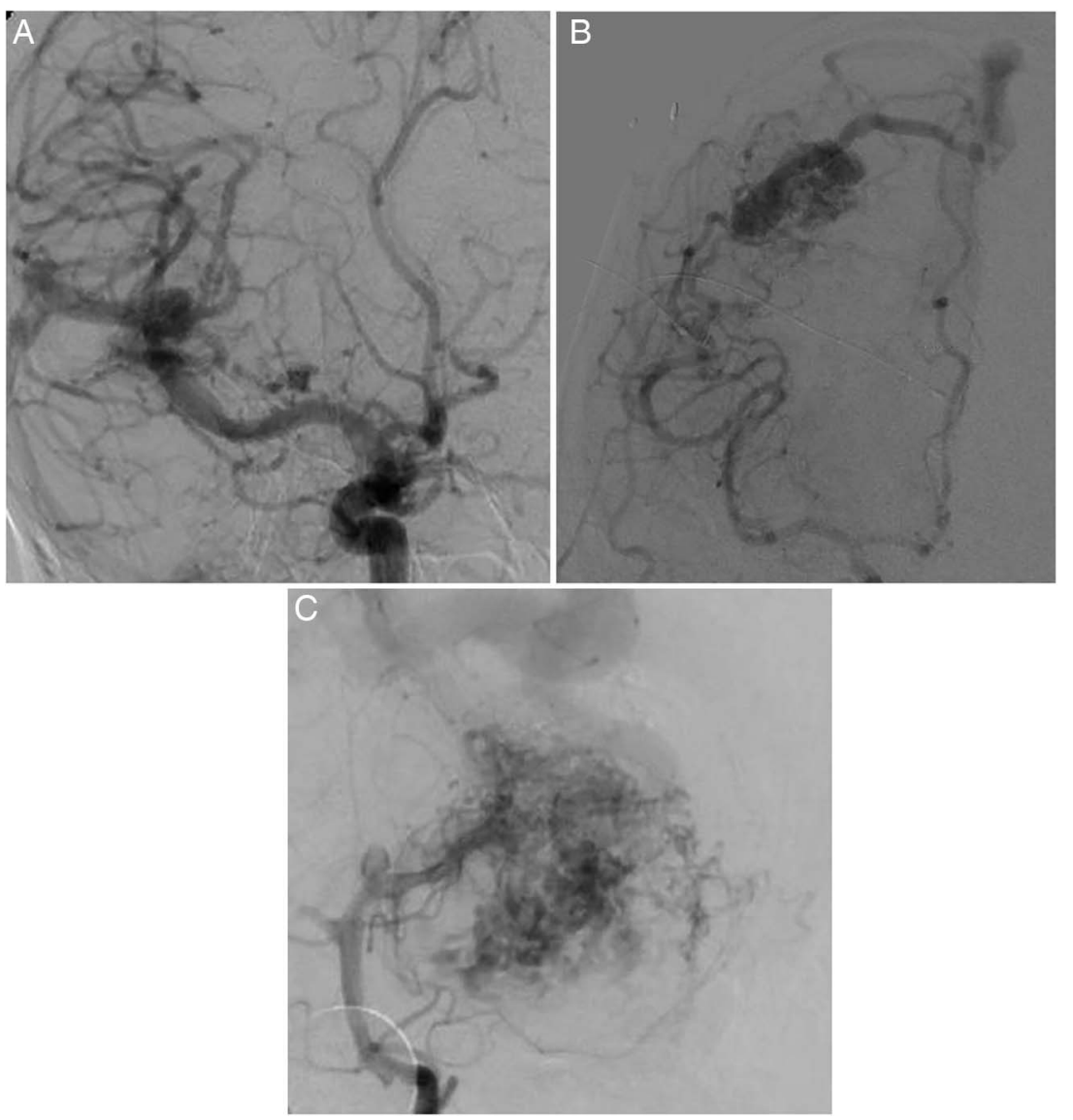
Figure 3 Example of size grading. The size of the nidus was measured and graded as 1 if it was $<3 \mathrm{~cm}(\mathrm{~A})$, 2 if it was $3-6 \mathrm{~cm} \mathrm{(B),} \mathrm{and} 3$ if it was $>6 \mathrm{~cm} \mathrm{(C).}$

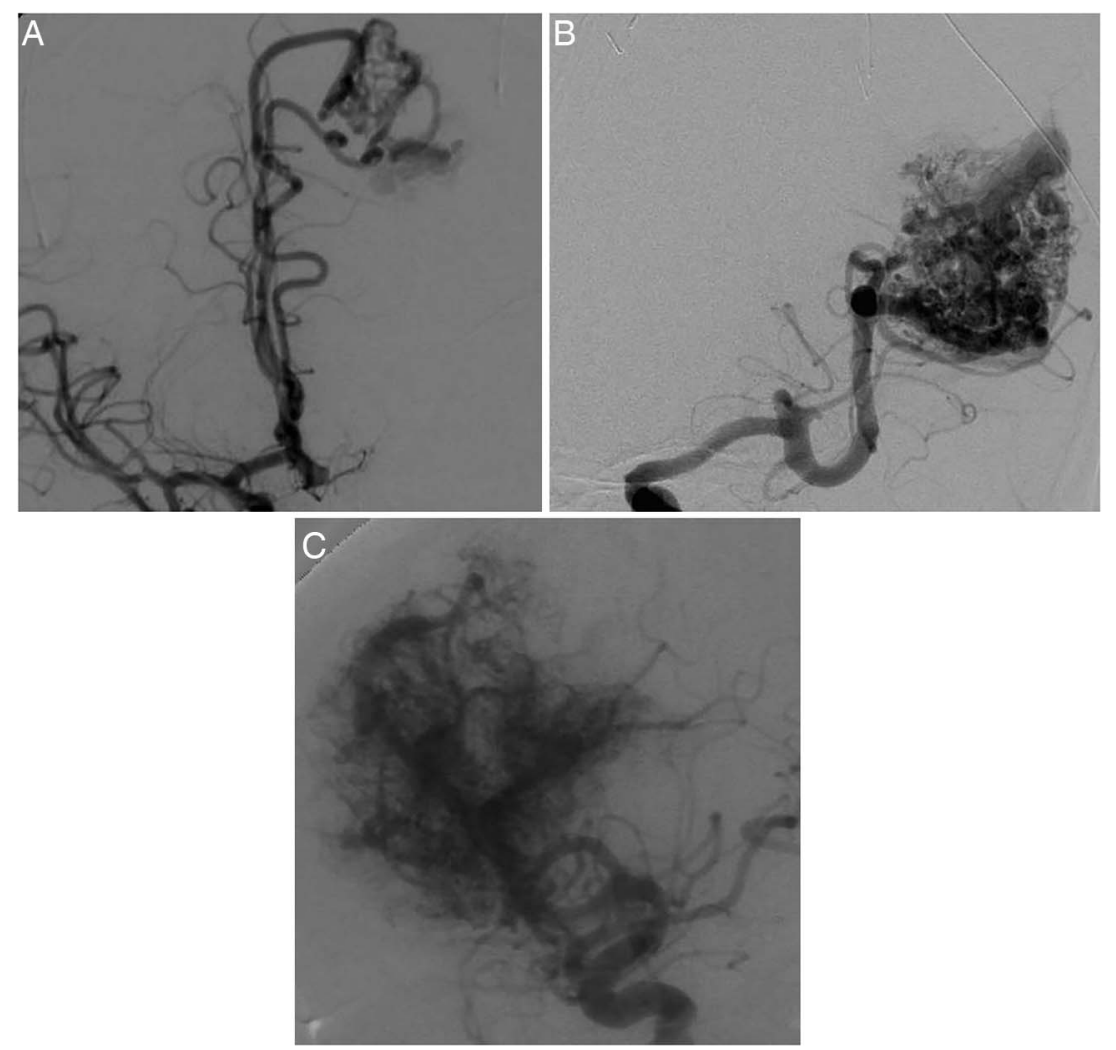

After identifying the angiographic factors that were more often associated with embocure we correlated these results with clinical outcome. With this information, we developed a simple and easily applied scoring system for each of these variables (table 1); the sum of these scores represents the total AVMES (figures 5 and 6).

\section{Study design and statistical analysis}

A retrospective cohort analysis of the AVMES was performed. The primary outcome measures were the rate of complete AVM obliteration and the incidence of major complications following embolization of cerebral AVMs with Onyx. Patients who had multiple embolization procedures were considered as a single case. In other words, the overall embolization risk to achieve complete angiographic embolization (ie, embocure) for each patient was considered. Patients were classified based on their AVMES. Rate of AVM obliteration and the risk of major complications were compared across different AVMES. A receiveroperator curve (ROC) analysis of the AVMES was performed to evaluate the characteristics of the score in predicting major complications due to AVM embolization. One-way analysis of variance was used to generate the test statistic for ordinal variables. Student's t test was used to compare the incidence of major complications between different groups. Statistical analysis was performed using STATA V.9.2 (College Station, Texas, USA).

\section{RESULTS}

Thirty-nine patients were identified who underwent primary endovascular Onyx embolization of their AVMs at our institution between 2005 and 2011. The average age of patients was 41 years (range 1-71). Fifty-one per cent of patients were female. Presenting symptoms were hemorrhage (intracranial hemorrhage or subarachnoid hemorrhage) in $23 \%$, seizures in $31 \%$, headaches in $21 \%$, visual symptoms in $15 \%$, paresis or numbness in $13 \%$, speech difficulties in $3 \%$. Thirteen per cent of patients had incidentally discovered AVMs (table 2).

Forty-eight per cent of the AVMs were located on the left side. Twenty-eight per cent were frontal, 21\% were parietal, $21 \%$ were temporal, $15 \%$ were occipital, $15 \%$ were in the posterior fossa, and $8 \%$ were in other locations.

Patients underwent an average of 1.6 (range 1-5) embolization procedures with curative intent. 1.5 (range 1-3) pedicles were accessed to inject liquid embolic material per patient. On average, $8.4 \mathrm{~mL}$ (range $0.2-59.98 \mathrm{~mL}$ ) of Onyx was injected

Complete AVM obliteration was attained in 67\% (26/39 patients). Major complications occurred in 13\% (5/39) patients. There was one $(2.6 \%)$ fatal re-hemorrhage after embolization that occurred in a patient who had an incomplete embolization and underwent postembolization stereotactic radiosurgery. Minor complications-including technical complications or changes in intraoperative evoked potentials without clinical consequence-occurred in $8 / 39$ patients $(20.5 \%)$. There were no access related complications. Fifty-nine per cent (23/39) achieved complete AVM obliteration without a major complication, 28\% (11/39) underwent an uncomplicated embolization course but did not achieve AVM obliteration, 3/39 (8\%) achieved AVM obliteration but sustained a major complication and 2/39 (5\%) sustained a major complication without achieving AVM obliteration (table 3). Good neurologic outcome (modified Rankin Score 0-3) was achieved in $84 \%$.

The AVMES was used to stratify these AVMs. There was a non-significant trend towards a greater number of pedicles accessed and used for embolization during the procedure with increasing AVMES. The volume of Onyx injected was significantly higher with higher AVMES. AVM obliteration rates were significantly higher with lower AVMES scores. Rates of AVM obliteration without complication were also significantly higher into the AVM nidus. 

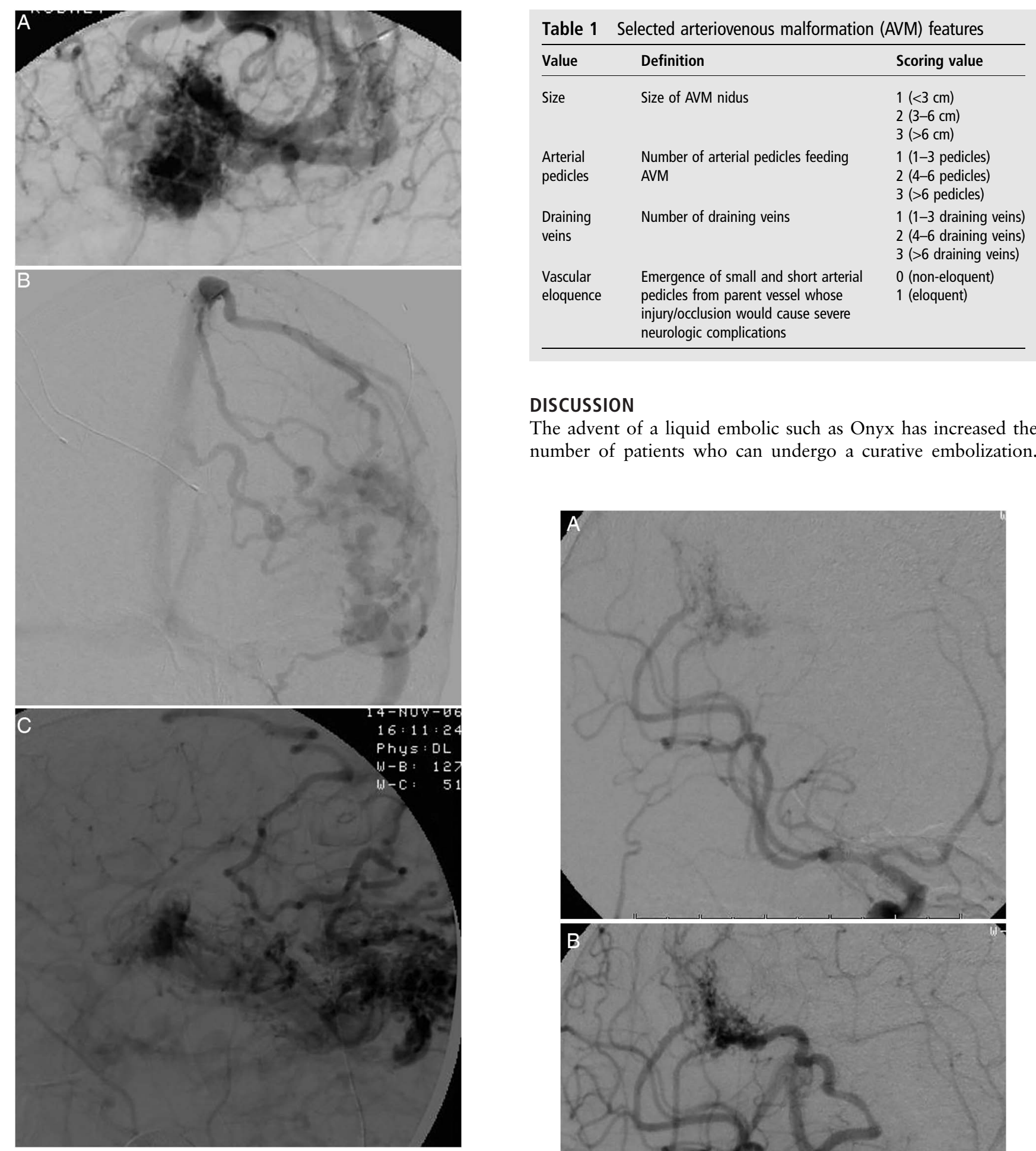

Figure 4 Example of grade of draining veins. An arteriovenous malformation was given a draining vein grade of 1 if $1-3$ draining veins were identified (A), 2 if $4-6$ draining veins were identified (B), and 3 if $>6$ draining veins were identified (C).

with lower AVMES (figure 7). Major complications increased with increasing AVMES (figure 8). Additionally, minor complication rates were greatest in the group with AVMES $>5$ (table 4). We performed a ROC analysis to quantify the predictive capacity of the endovascular AVM score. For complete AVM obliteration, the AVMES had an area under the curve (AUC) of 0.8240 (95\% CI 0.6897 to 0.9582 ). For major complications, the AVMES had an AUC 0.7529 (95\% CI 0.54105 to 0.96483 ).
Table 1 Selected arteriovenous malformation (AVM) features

\begin{tabular}{lll}
\hline Value & Definition & Scoring value \\
\hline Size & Size of AVM nidus & $1(<3 \mathrm{~cm})$ \\
& & $2(3-6 \mathrm{~cm})$ \\
& & $3(>6 \mathrm{~cm})$ \\
Arterial & Number of arterial pedicles feeding & $1(1-3$ pedicles $)$ \\
pedicles & AVM & $2(4-6$ pedicles) \\
& & $3(>6$ pedicles $)$ \\
Draining & Number of draining veins & $1(1-3$ draining veins) \\
veins & & $2(4-6$ draining veins) \\
& & $3(>6$ draining veins) \\
Vascular & Emergence of small and short arterial & 0 (non-eloquent) \\
eloquence & pedicles from parent vessel whose & 1 (eloquent) \\
& injurylocclusion would cause severe & \\
& neurologic complications &
\end{tabular}

\section{DISCUSSION}

The advent of a liquid embolic such as Onyx has increased the number of patients who can undergo a curative embolization.

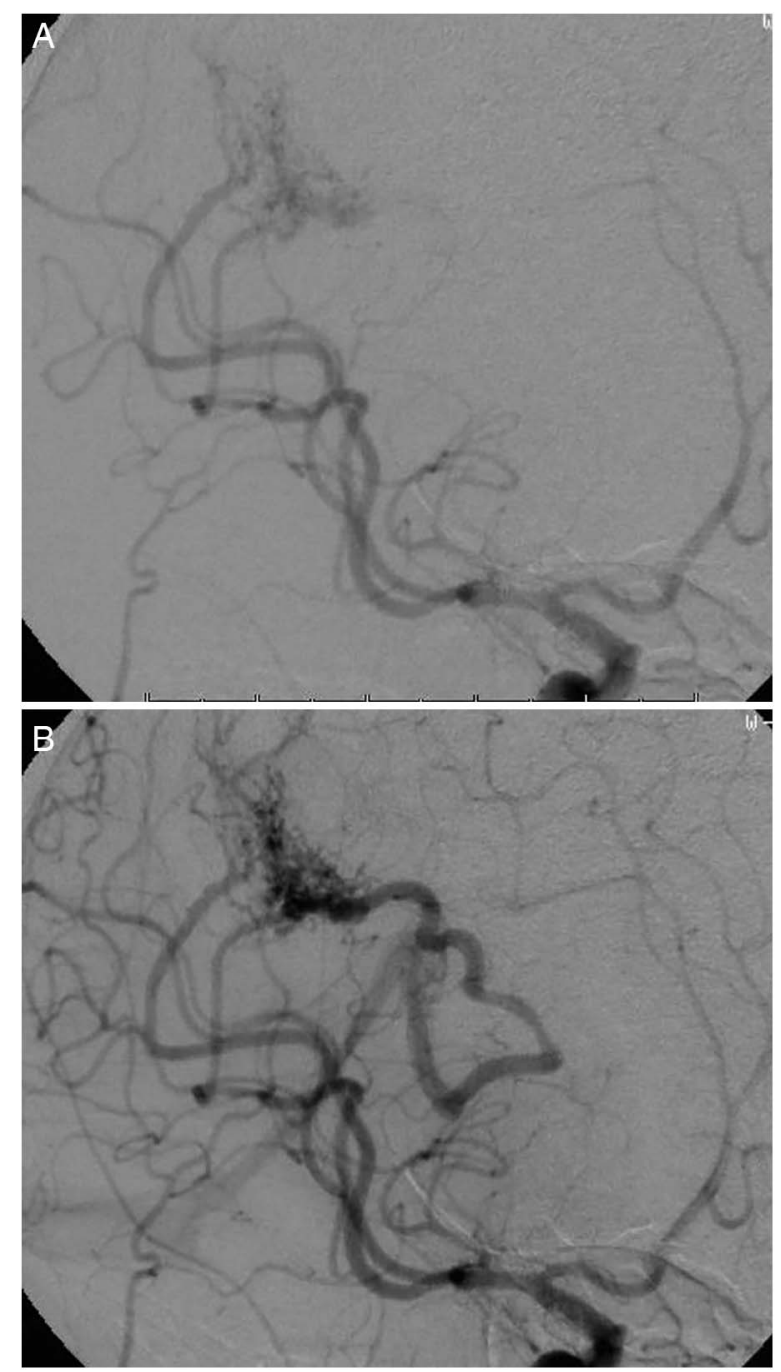

Figure 5 Early (A) and mid- (B) arterial phases, anteroposterior views of carotid cerebral angiogram demonstrating an arteriovenous malformation (AVM) embocure score (AVMES) of 4 AVM. This AVM is $<3 \mathrm{~cm}$ in diameter (1 point), drains through $<4$ veins (1 point), has 4 clearly identifiable arterial pedicles ( 2 points), and does not meet the criteria for eloquence (0 points). 


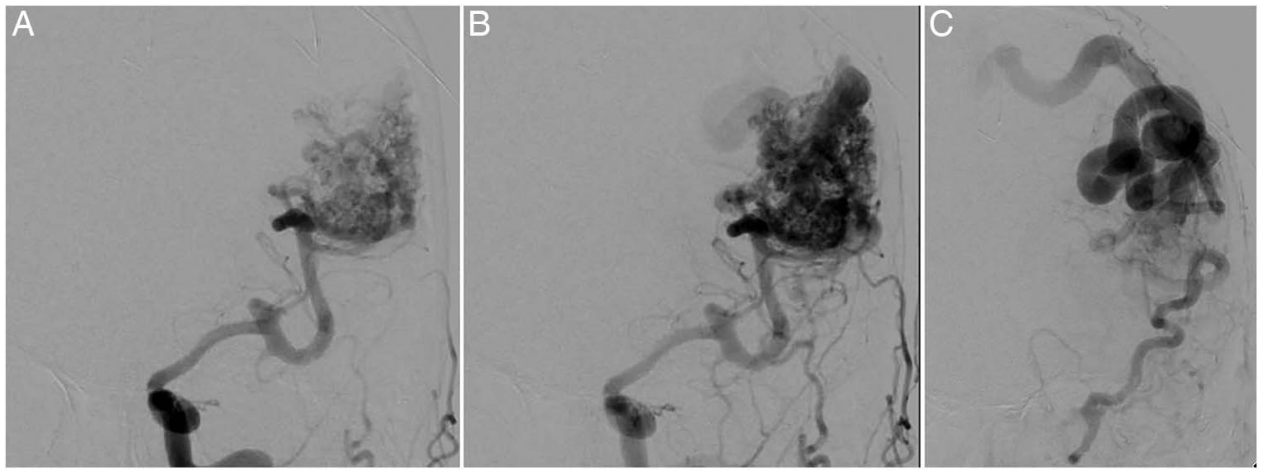

Figure 6 Early (A) and mid- (B) arterial and venous (C) phases, anteroposterior views of carotid cerebral angiogram demonstrating an arteriovenous malformation (AVM) embocure score (AVMES) 5 AVM. This AVM is about $5 \mathrm{~cm}$ in diameter ( 2 points), drains through $<4$ veins (1 point), has <4 clearly identifiable arterial pedicles (1 point), and meets criteria for eloquence (1 point).

This is in large part due to the cohesive nature of Onyx (compared with the adhesive nature of N-butyl cyanoacrylate (n-BCA)), which avoids adherence of the embolic material to the microcatheter tip and thus enables longer working times and, consequently, a more controlled and complete embolization. ${ }^{11}$ While endovascular embolization of AVMs traditionally has been an adjuvant treatment, or used in the context of multimodal therapy, it is increasingly possible to achieve complete radiographic AVM obliteration through embolization procedures alone. $^{12-15}$ The increasing number of case series demonstrating radiographic cures via endovascular embolization further extends the already complex therapeutic landscape of AVM treatment.

Endovascular embolization as a curative treatment is in its early phases. Traditionally, the literature has considered an AVM to be cured when there is no radiographic evidence of AVM filling. However, when applying this definition to curative endovascular embolization, the longevity of this treatment remains to be defined. Though, in our series, we did not observe any bleeding/rebleeding in patients who we consider achieved curative endovascular embolization, we apply the term 'curative' with caution and understand that long-term follow-up of these patients is required. It is unclear how long a patient needs to be followed up with imaging after a complete embolization of an AVM. Currently, our protocol is MRI/MRA with contrast and a six-vessel DSA performed at 6 months after embolization to consider complete occlusion of the AVM. At this point, we recommend yearly MRI/MRA after confirmation with DSA and MRI/MRA that the AVM is occluded. Once more data are

Table 2 Demographics and presentation

\begin{tabular}{llll}
\hline & Mean & $95 \% \mathrm{CI}$ & \\
\hline Age (years) & 44.1 & 38.6 & 49.6 \\
Female (\%) & 51 & 35 & 68 \\
Presenting signs/symptoms (\%) & & & \\
ICH/SAH & 23 & 0 & 27 \\
Seizures & 31 & 16 & 46 \\
Headaches & 21 & 7 & 34 \\
Visual disturbance & 15 & 4 & 27 \\
Weakness/numbness & 13 & 2 & 24 \\
Speech difficulty & 3 & 0 & 8 \\
Incidental & 13 & 2 & 24 \\
\hline ICH, intracranial hemorrhage; SAH, subarachnoid hemorrhage.
\end{tabular}

\begin{tabular}{llll}
\hline & Mean (\%) & \multicolumn{2}{c}{$95 \% \mathrm{Cl}$} \\
\hline Complications (\%) & & & \\
$\quad$ Major & 13 & 2 & 24 \\
$\quad$ Minor & 21 & 7 & 34 \\
Outcomes (\%) & & & \\
$\quad$ AVM obliteration (overall) & 67 & 51 & 82 \\
Uncomplicated AVM obliteration & 59 & 43 & 75 \\
Uncomplicated embolization, residual AVMs & 28 & 13 & 43 \\
AVM obliteration with major complication & 8 & 0 & 16 \\
$\quad$ Major complication, residual AVMs & 5 & 0 & 12 \\
\hline AVM, arteriovenous malformation. & & &
\end{tabular}

Table 3 Overall complications and outcomes

available about the durability of this treatment we may be able to establish a minimum level of long-term imaging follow-up with more confidence.

The Spetzler-Martin scale and its recent modifications ${ }^{16}$ provide a means of risk stratification for the microsurgical management of AVMs, whereas the AVM score provides a riskstratification tool for radiosurgical management. However, to date, there has been no risk-stratification tool for AVMs that incorporates the specific angioarchitectural features that are particularly relevant to the endovascular treatment of AVMs. We proposed the AVMES and applied it to a cohort of patients who were treated with curative intent using Onyx embolization.

Starke et $a l^{17}$ evaluated their experience with n-BCA and found that small and large size, eloquent location, deep venous drainage, and complex vascular anatomy requiring multiple embolization procedures were risk factors for the development of postprocedural neurologic deficit. Although some of these elements mirror those of the AVMES proposed in our study, one must exercise caution in attempting to extrapolate these risk factors to procedures using Onyx given the inherent differences between n-BCA and Onyx. The improved control and direction associated with the use of Onyx may make its morbidity risk factors unique.

Recently, Dumont et $a l^{18}$ proposed a grading system for the endovascular treatment of AVMs. They applied their proposed scale to 50 patients treated endovascularly, including treatment with Onyx and n-BCA. As mentioned, given the inherent differences between the two agents, factors attributing risk to n-BCA may be obviated simply by using Onyx. Additionally, the

AVM, arteriovenous malformation. 


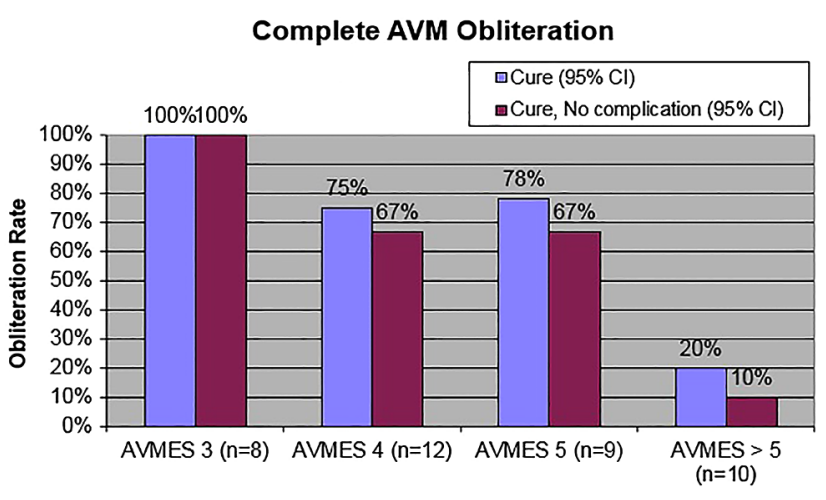

AVMES

Figure 7 Rates of complete arteriovenous malformation (AVM) obliteration according to AVM embocure score (AVMES).

AVMES differs from the system reported by Dumont et al in its consideration of the venous drainage and the introduction of the concept of 'vascular eloquence'. Though venous drainage may be more important during microsurgical treatment of AVMs, recognition of the nidal outflow patterns remains critical when considering endovascular therapy.

The concept of 'vascular eloquence' is unique to the AVMES. When performing endovascular embolization, we believe this 'vascular eloquence' to be of greater importance than traditionally described brain eloquence since a proximal, parent vessel occlusion would result in significant, often hemispheric, deficits. This is in contrast to reflux into a distal, cortical parent vessel, which may cause isolated focal deficits. Additionally, the use of Wada testing during endovascular procedures can assess the potential deficits in a given eloquent region.

We found the AVMES to be a robust tool in structuring the risk-benefit assessment of obliterative embolization of a particular AVM. Its strengths are multifold. First, similar to the SpetzlerMartin scale, it is easily applied and simple to calculate. Second, its components are intuitively important to endovascular surgeons experienced in AVM embolization. Specifically:

- Complications tend to increase with the number of arterial pedicles accessed.

- Reflux of embolic material into parent arteries are much more prohibitive events when occurring in proximal vessels that supply large and/or critical brain regions.

- The greater the size of the nidus the longer the procedure and the greater the volume of embolic material required for obliteration. These factors may increase the chance of complication or abortion of the procedure.

\section{Major Post-Procedural Complications}

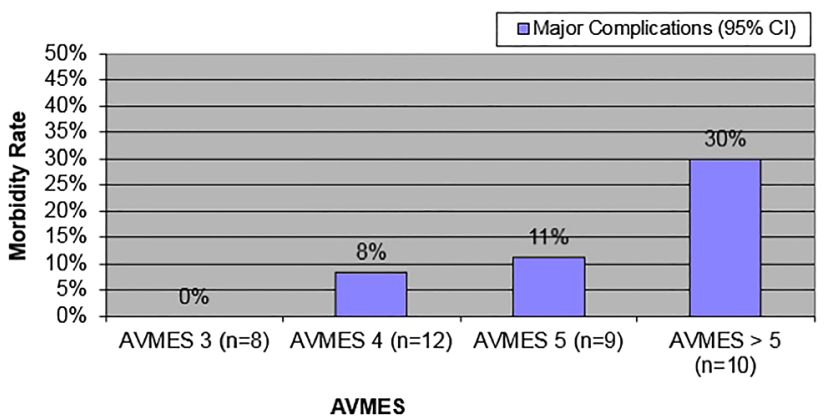

Figure 8 Rates of major postprocedural complication according to arteriovenous malformation embocure score (AVMES).
- Since the venous compartment of the AVM also must be occluded for radiographic cure, in a way similar to the number of arterial pedicles, the greater the number of draining veins, the more difficult it is to achieve this task.

Finally, as we demonstrate here, the AVMES has significant discriminatory power in distinguishing AVMs that are at low, intermediate, and high risk of embocure success. The application of the AVMES to assess the likelihood of curative embolization distinguishes our study from previous proposals that examined only the risk of neurologic complication.

Specifically, we found that patients with AVMs who have an AVMES of 3 have a favorable risk profile for primary endovascular obliteration of their AVM (100\% obliteration rate without complication). Patients with a score of 4 or 5 have similar risk profiles, with $67 \%$ achieving complete obliteration without complication. On the contrary, we found that patients with AVMES $>5$ were a high-risk embolization group. These patients most frequently (60\% of the time) had partial embolizations without complication, but only $10 \%$ achieved complete obliteration without complication. Moreover, 30\% of these patients had major complications.

Our complication rate is in line with a meta-analysis of cerebral AVM treatment outcomes by modality. ${ }^{19}$ In that meta-analysis, embolization carried a $6.6 \%$ median risk of severe complication (range 0-28\%), stereotactic radiosurgery carried a 5.1\% median risk of severe complication (range 0-21\%), and microsurgery carried a $7.4 \%$ median risk of severe complication (range 0$40 \%$ ). Our obliteration rate compares favorably with previously published series, where the median obliteration rate of AVM embolization was 13\% (range 0-94\%). For radiosurgery median obliteration rates were $38 \%$ (range $0-75 \%$ ) and for microsurgery median obliteration rates were 96\% (range 0-100\%).

Our ROC analysis demonstrated that application of the endovascular AVMES provides good discriminatory power in evaluating AVM obliteration rates (both overall-AUC $=0.8240$ - and obliteration rates without complication-AUC $=0.8356$ ). It performed well, but was less robust, in discriminating cases with major complications (AUC $=0.7529)$.

The recent publication of the ARUBA trial ${ }^{20}$ - with early results favoring observation over intervention rather than any form of treatment (embolization, microsurgery, or radiosurgery) in the management of AVMs-has made tools that may improve our patient selection in treating AVMs increasingly pertinent. We hope that further refinement in patient and treatment modality for AVMs through the use of clinical tools such as the AVMES may improve our ability to provide the best personalized care for patients with this challenging disease.

This study represents an origination (training) dataset for the generation of the AVMES and suffers from the limitations and biases inherent to this type of dataset. External validation in a separate dataset is needed to confirm the generalizability of our scoring system. Our study included 39 patients, with about 10 patients in each group. This sample size, with its resultant effect on power, may represent a limitation of the study. We hope to validate this scale further through its application in a prospective manner. Another limitation is that AVMES was designed based on experience only with Onyx as the embolic material and no balloon or detachable microcatheters were used. This new technology may assist in increasing higher rates of embocure but this is yet to be shown.

In our study, we consider observed complete embolization to be curative. We applied the AVMES to DSA performed at 6 months' follow-up. Our study lacks long-term data and the possibility of recurrence after complete embolization seen at 
Table 4 Procedural features, complications, and outcomes by AVMES

\begin{tabular}{|c|c|c|c|c|c|c|c|c|c|c|c|c|c|}
\hline & \multicolumn{3}{|c|}{ AVMES 3} & \multicolumn{3}{|c|}{ AVMES 4} & \multicolumn{3}{|c|}{ AVMES 5} & \multicolumn{3}{|c|}{ AVMES > 5} & \multirow{2}{*}{$\begin{array}{l}\text { ANOVA } \\
\text { p Value }\end{array}$} \\
\hline & Mean & $95 \% \mathrm{Cl}$ & & Mean & $95 \% \mathrm{Cl}$ & & Mean & $95 \% \mathrm{Cl}$ & & Mean & $95 \% \mathrm{Cl}$ & & \\
\hline Number of embolization procedures & 1.38 & 0.75 & 2.00 & 1.50 & 0.93 & 2.07 & 1.44 & 0.67 & 2.22 & 2.10 & 1.18 & 3.02 & 0.3835 \\
\hline Number of pedicles injected $(n=38)$ & 1.13 & 0.83 & 1.42 & 1.33 & 0.92 & 1.75 & 1.50 & 1.05 & 1.95 & 1.90 & 1.19 & 2.61 & 0.1198 \\
\hline Volume of Onyx injected (mL, $n=30)$ & 1.97 & 0.48 & 3.46 & 5.12 & 0.00 & 12.46 & 4.59 & 0.63 & 8.54 & 25.21 & 3.79 & 46.63 & 0.0033 \\
\hline \multicolumn{14}{|l|}{ Complications (\%) } \\
\hline Major & 0 & 0 & 0 & 8 & 0 & 27 & 11 & 0 & 37 & 30 & 0 & 65 & 0.2727 \\
\hline Minor & 25 & 0 & 64 & 17 & 0 & 41 & 0 & 0 & 0 & 40 & 3 & 77 & 0.1933 \\
\hline \multicolumn{14}{|l|}{ Outcomes (\%) } \\
\hline AVM obliteration (overall) & 100 & 100 & 100 & 75 & 46 & 100 & 78 & 44 & 100 & 20 & 0 & 50 & 0.0008 \\
\hline Uncomplicated AVM obliteration & 100 & 100 & 100 & 67 & 35 & 98 & 67 & 28 & 100 & 10 & 0 & 33 & 0.0003 \\
\hline Uncomplicated embolization, residual AVMs & 0 & 0 & 0 & 25 & 0 & 54 & 22 & 0 & 56 & 60 & 23 & 97 & 0.0358 \\
\hline AVM obliteration with major complication & 0 & 0 & 0 & 8 & 0 & 27 & 11 & 0 & 37 & 10 & 0 & 33 & 0.8433 \\
\hline Major complication, residual AVMs & 0 & 0 & 0 & 0 & 0 & 0 & 0 & 0 & 0 & 20 & 0 & 50 & 0.1092 \\
\hline
\end{tabular}

6 months' follow-up is not captured in this study. As mentioned, we recognize that application of this score to long-term follow-up is necessary to confirm its validity and durability.

We believe that the AVMES will provide endovascular surgeons with an invaluable tool for predicting the likelihood of a successful radiographic cure, as well as enabling a risk-benefit assessment of AVMs treated with Onyx endovascular embolization. This is much needed when evaluating patients with cerebral arteriovenous malformations. When used in conjunction with the Spetzler-Martin scale and the AVM score, this tool may best guide physicians in determining a treatment modality with the most favorable risk-benefit ratio.

\section{CONCLUSION}

No formal grading system is currently available to aid in the risk assessment of endovascular embolization of AVMs with Onyx. We present the endovascular AVM score to fulfill the need for such a tool. The endovascular AVM score provides a simple, intuitive, easily assessed scoring system that robustly discriminates between groups with favorable, intermediate, and unfavorable risk profiles to achieve a curative endovascular embolization. Though validation prospectively and with long-term follow-up is needed, we hope this tool will aid in the assessment and therapeutic decision-making of microsurgeons, radiosurgeons, and endovascular surgeons in their management of AVMs.

Contributors DKL, RM, DS and SM were involved in the study concept, design, data analysis, manuscript writing, and review. FC and MCK were involved in data collection and analysis.

Competing interests DKL has financial and research relationships with Covidien, Stryker, and Penumbra. RM is a consultant for Covidien.

Provenance and peer review Not commissioned; externally peer reviewed.

\section{REFERENCES}

1 Spetzler RF, Martin NA. A proposed grading system for arteriovenous malformations. J Neurosurg 1986;65:476-83.

2 Nataf $F$, Schlienger $M$, Bayram $M$, et al. Microsurgery or radiosurgery for cerebral arteriovenous malformations? A study of two paired series. Neurosurgery 2007;61:39-50.

3 Porter PJ, Shin AY, Detsky AS, et al. Surgery versus stereotactic radiosurgery for small, operable cerebral arteriovenous malformations: a clinical and cost comparison. Neurosurgery 1997;41:757-66.
4 Lišcák R, Vladyka V, Šimonová G, et al. Arteriovenous malformations after Leksell gamma knife radiosurgery: rate of obliteration and complications. Neurosurgery 2007;60:1005-16.

5 Starke RM, Komotar RJ, Hwang BY, et al. Treatment guidelines for cerebral arteriovenous malformation microsurgery. Br J Neurosurg 2009;23:376-86.

6 Weber W, Kis B, Siekmann R, et al. Endovascular treatment of intracranial arteriovenous malformations with onyx: technical aspects. Am J Neuroradiol 2007:28:371-7.

7 Hartmann A, Pile-Spellman J, Stapf $C$, et al. Risk of endovascular treatment of brain arteriovenous malformations. Stroke 2002;33:1816-20.

8 Andrade-Souza YM, Zadeh G, Ramani M, et al. Testing the radiosurgery-based arteriovenous malformation score and the modified Spetzler-Martin grading system to predict radiosurgical outcome. J Neurosurg 2005; 103:642-8.

9 Pikus HJ, Beach ML, Harbaugh RE. Microsurgical treatment of arteriovenous malformations: analysis and comparison with stereotactic radiosurgery. J Neurosurg 1998;88:641-6.

10 Pollock BE, Flickinger JC. A proposed radiosurgery-based grading system for arteriovenous malformations. J Neurosurg 2002;96:79-85.

11 Loh Y, Duckwiler GR. A prospective, multicenter, randomized trial of the Onyx liquid embolic system and N-butyl cyanoacrylate embolization of cerebral arteriovenous malformations. J Neurosurg 2010;113:733-41.

12 Katsaridis V, Papagiannaki C, Aimar E. Curative embolization of cerebral arteriovenous malformations (AVMs) with Onyx in 101 patients. Neuroradiology 2008;50:589-97.

13 Reig AS, Rajaram R, Simon S, et al. Complete angiographic obliteration of intracranial AVMs with endovascular embolization: incomplete embolic nidal opacification is associated with AVM recurrence. J Neurointerv Surg 2010; 2:202-7.

14 Andreou A, loannidis I, Lalloo S, et al. Endovascular treatment of intracranial microarteriovenous malformations: clinical article. J Neurosurg 2008;109:1091-7.

15 Panagiotopoulos V, Gizewski E, Asgari S, et al. Embolization of intracranial arteriovenous malformations with ethylene-vinyl alcohol copolymer (Onyx). Am J Neuroradiol 2009;30:99-106

16 Rodriguez-Hernandez A, Kim H, Pourmohamad T, et al. Cerebellar arteriovenous malformations: anatomic subtypes, surgical results, and increased predictive accuracy of the supplementary grading system. Neurosurgery 2012;71:1111-24.

17 Starke RM, Komotar RJ, Otten ML, et al. Adjuvant embolization with N-butyl cyanoacrylate in the treatment of cerebral arteriovenous malformations: outcomes, complications, and predictors of neurologic deficits. Stroke 2009;40:2783-90.

18 Dumont T, Kan P, Snyder K, et al. A proposed grading system for endovascular treatment of cerebral arteriovenous malformations: Buffalo score. Surg Neurol Int 2015;6(1):3.

19 van Beijnum J, van der Worp HB, Buis DR, et al. Treatment of brain arteriovenous malformations: a systematic review and meta-analysis. JAMA 2011;306:2011-19.

20 Mohr J, Parides MK, Stapf C, et al. Medical management with or without interventional therapy for unruptured brain arteriovenous malformations (ARUBA): a multicentre, non-blinded, randomised trial. Lancet 2014;383:614-21. 